\title{
Communications
}

\section{Photoinduced Fluorescence "Turn-On" Micropatterning with Substituted Triphenylmethane Derivatives}

\author{
Junwoo Kim, Sumi Lee, and Jong-Man Kim* \\ Department of Chemical Engineering, Hanyang University, Seoul 133-791, Korea. *E-mail: jmk@hanyang.ac.kr \\ Received August 10, 2010, Accepted August 19, 2010
}

Key Words: Fluorescence patterning, Triphenylmethane, UV irradiation

\begin{abstract}
Methods for the generation of patterned fluorescence images based on the "precursor approach" has gained great attention recently. ${ }^{1}$ The "precursor approach" enables rapid and costeffective generation of patterned images without the need for additional wet developing processes. Conceptually, the 'precursor approach' is based on the use of a polymer containing a dye molecule that has different excited state electronic properties before and after external stimulation. ${ }^{2-5}$ This approach is exemplified by a system comprised of a dye molecule that is nonfluorescent (or fluorescent) when the key functional group of the dye molecule is protected with a protecting group. Photoinduced selective removal of the protecting group then produces an image that is fluorescent (nonfluorescent). The acid labile tert-butoxycarbonyl group ( $t$-Boc) is commonly employed as the protecting group in systems of this type since it can be readily removed by UV irradiation in the presence of a photoacid generators (PAG). ${ }^{6,7}$
\end{abstract}

Photoinduced oxidation of a precursor molecule can be employed to generate patterned fluorescence images in polymer films. ${ }^{8-10}$ This strategy takes advantage of the fact that certain fluorescent compounds often lose their ability to fluoresce when they undergo UV-induced oxidative degradation. This photoinduced fluorescence quenching strategy ("Turn-Off" strategy), in conjunction with fluorescent polymers or polymers with pendant fluorescent chromophores, has been used for fluorescence patterning.

In the course of studies aimed at the development of precursor molecules that can be used to produce patterned fluorescence images in the polymer films, we discovered that electronrich triphenylmethane derivatives serve as efficient substances in methods for "Turn-On"-type fluorescent image formation. It is well known photoinduced oxidation of electron donating group-substituted triphenylmethane derivatives produces brilliantly colored ionic species (Scheme 1). ${ }^{11}$ Owing to this property, triphenylmethane derivatives have been investigated as precursors of colorants. In our efforts, we observed that photoinduced color forming reactions of triphenylmethane derivatives is also accompanied by the generation of fluorescence. The photoinduced fluorescence of triphenylmethane derivatives appears to be significant in light of its potential application to the generation of "Turn-On"-type patterned fluorescence images in the polymer films. It should be noted that, in general, it is easier to observe patterned fluorescence images when non-

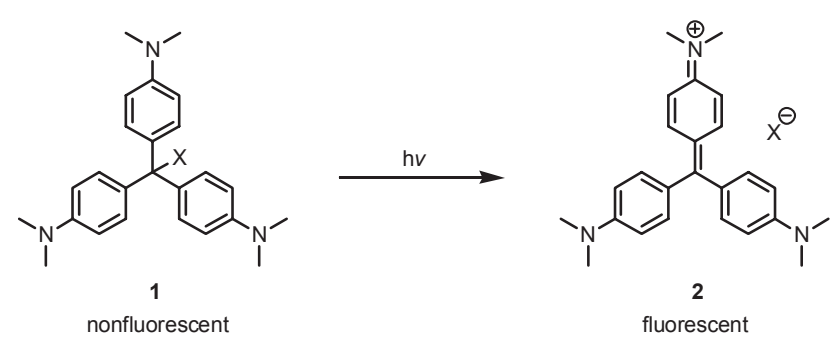

Scheme 1. Schematic of the precursor method for generation of patterned fluorescence images
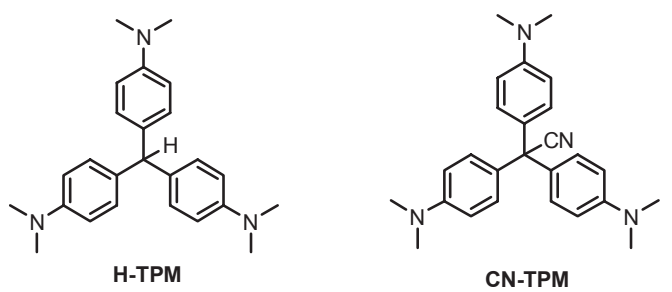

Scheme 2. Structures of triphenylmethane-derived precursor molecules

fluorescent precursor molecule generate the fluorescent products (i.e., "Turn-On"-type).

In order to test above mentioned feasibility of fluorescence "Turn-On" patterning, two triphenylmethane derivatives 4,4', 4"-methanetriyltris( $N, N$-dimethylaniline) (H-TPM) ${ }^{12}$ and 2, 2,2tris(4-(dimethylamino)phenyl)acetonitrile (CN-TPM) ${ }^{11}$ and were investigated as precursor molecules.

Initial phase this effort focused on the fabrication of patterned fluorescence images using H-TPM. A thin poly(methyl meth-

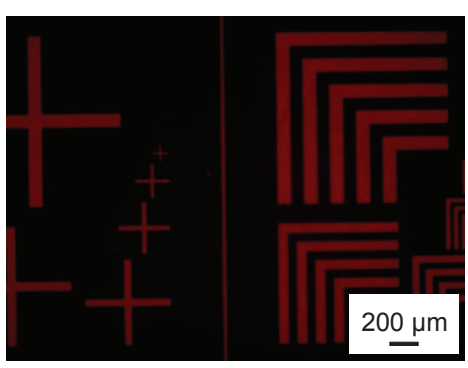

Figure 1. Patterned fluorescence images obtained by a photomasked UV irradiation $\left(254 \mathrm{~nm}, 12 \mathrm{~mW} / \mathrm{cm}^{2}, 30 \mathrm{~s}\right)$ of a $c a .1 \mu \mathrm{m}$ thick PMMA (80 wt \%) film containing the precursor H-TPM $(20 \mathrm{wt} \%)$. 
<smiles>Cc1ccc(C(c2ccc(N(C)C)cc2)(c2ccc(N(C)C)cc2)c2ccc(N(C)C)cc2)cc1</smiles><smiles>CN(C)c1ccc(C(c2ccccc2)(c2ccc(N(C)C)cc2)c2ccc(N(C)C)cc2)cc1</smiles>

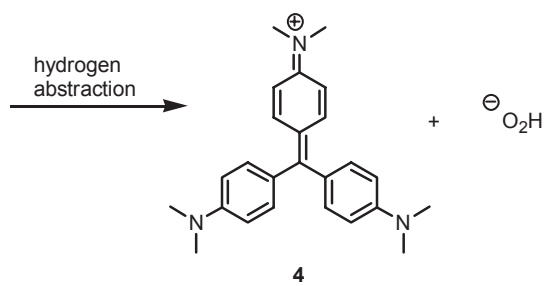

Scheme 3. Proposed mechanism of photoinduced iminium ion formation from H-TPM

acrylate) (PMMA) film (ca. $1 \mu \mathrm{m})$ on a glass substrate was prepared by spin-coating a chloroform solution containing H-TPM. The film was irradiated with UV light $(254 \mathrm{~nm}, 12 \mathrm{~mW} /$ $\mathrm{cm}^{2}$ ) for $30 \mathrm{~s}$ through a photomask. In Figure 1 is shown the patterned fluorescence images under a fluorescence microscope. Although the patterned images are detectable, their resolution is relatively low.

The mechanism responsible for photoconversion of H-TPM to the key iminium ion species $\mathbf{4}$, which serves as the fluorescent moiety in this system, is shown in Scheme 3. The first step of this process involves excited state single electron transfer (SET) from the triphenylmethane donor to presumably molecular oxygen. The radical cation intermediate $\mathbf{3}$, produced in this fashion, subsequently undergoes hydrogen atom loss to yield the iminium ion 4.

According to the mechanistic analysis given in Scheme 3, the poor resolution of the images generated from H-TPM is likely the result of the efficiency of hydrogen atom loss from the radical cation $\mathbf{3}$. It is well known that cyano-substituted triphenylmethane derivatives undergo rapid SET-photoinduced cleavage reactions to produce the corresponding iminium ions. ${ }^{11}$ This is likely the consequence of efficient homolytic cleavage of the cyano-methine carbon bond in cation radicals produced by initial excited state SET. In order to take advantage of this property, a cyano-substituted triphenylmethane CN-TPM, was investigated as a precursor alternative.

Photomasked UV irradiation of a thin polymer film ( $c a .1 \mu \mathrm{m}$

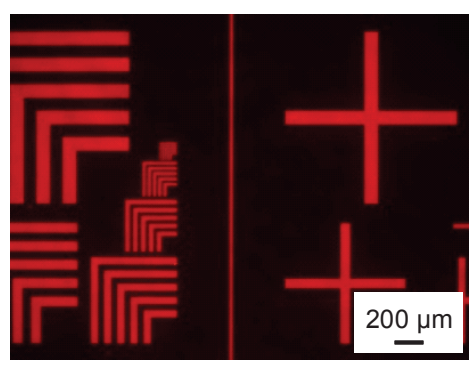

Figure 2. Patterned fluorescence images obtained by a photomasked UV irradiation $\left(254 \mathrm{~nm}, 12 \mathrm{~mW} / \mathrm{cm}^{2}, 30 \mathrm{sec}\right)$ of a $\mathrm{ca}$. 1 um thick PMMA (80 wt \%) film containing the precursor CN-TPM (20 wt \%).

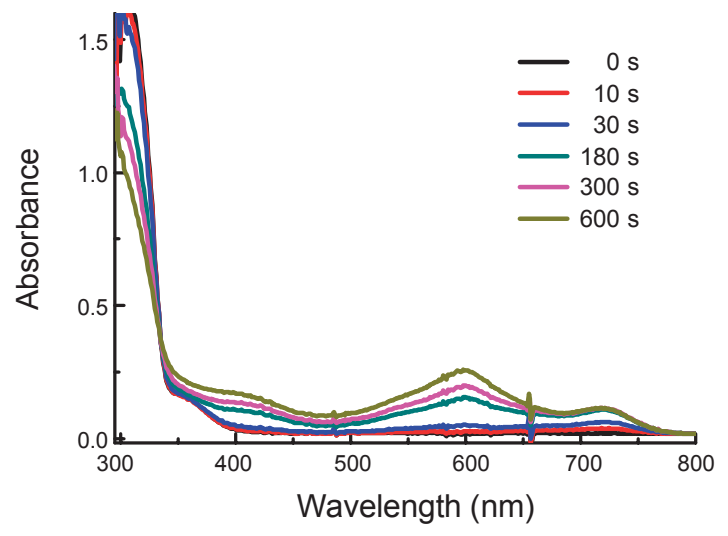

Figure 3. UV-visible absorption spectral change obtained with PMMA film containing the precursor molecule CN-TPM upon UV irradiation.

thickness), prepared with CN-TPM and PMMA on a glass substrate results in the clean generation of patterned fluorescence images (Figure 2). The bright red areas are the regions exposed to UV light. The quality of the fluorescence patterns is much higher in contrast to those produced using H-TPM.

Formation of the iminium ion species was confirmed by UV-visible spectroscopy (Figure 3). As displayed in Figure 3, $254 \mathrm{~nm}$ UV irradiation of a PMMA film containing the cyanide substituted triphenylmethane precursor molecule CN-TPM results in the generation of absortion bands in the visible region.

In summary, a fluorescence "Turn-On" approach was developed for patterned fluorescence images in spin-coated polymer film. The methodology employs substituted triphenylmethane derivatives as the precursor molecules. Photomasked irradiation of the film containing the precursor molecules with UV light $(254 \mathrm{~nm})$ afforded patterned fluorescence images. Among two compounds investigated, $\mathrm{CN}$-substituted precursor molecule CN-TPM yielded better image patterns over H-TPM presumably due to enhanced photooxydation property.

Acknowledgments. This work was supported by Research Foundation of Korea (KRF-2007-313-D00208).

\section{References and Notes}

1. For recent review, see: Kim, J.-M. Macromol. Rapid Commun. 2007, 28, 1191.

2. Lim, S.-J.; Seo, J.; Park, S. Y. J. Am. Chem. Soc. 2006, 128, 14542.

3. Kwak, G.; Lee, W.-E.; Kim, W.-H.; Lee. H. Chem. Commun. 2009 , 2112.

4. Lee, J. K.; Kim, H.-J.; Kim, T. H.; Lee, C.-H.; Park, W. H.; Kim, J.; Lee, T. S. Macromolecules 2005, 38, 9427.

5. Cho, S.-Y.; Song, Y.-K.; Kim, J.-G.; Oh, S.-Y.; Chung, Chan.-M. Tetrahedron Lett. 2009, 50, 4769.

6. Kim, J.-M.; Kang, J.-H.; Han, D.-K.; Lee, C.-W.; Ahn, K.-D. Chem. Mater. 1998, 10, 2332.

7. Kim, J.-M.; Chang, T.-E.; Kang, J.-H.; Han, D. K.; Ahn, K.-D. Adv. Mater. 1999, 11, 1499.

8. Lam, J. W. Y.; Qin. A.; Dong, Y.; Lai, L. M.; Häussler, M.; Dong, Y.; Tang, B. Z. J. Phys. Chem. B 2006, 110, 21613.

9. Kwak, G.; Fujiki, M.; Sakaguchi, T.; Masuda, T. Macromolecules 2006, 39, 319 .

10. Ohshita, J.; Uemura, T.; Kim, D.-H.; Kunai, A.; Kunugi, Y.; Kakimoto, M. Macromolecules 2005, 38, 730.

11. Malpert, J. H.; Grinevich, O.; Strehmel, B.; Jarikov, V.; Mejiritski, A.; Neckers, D. C. Tetrahedron 2001, 57, 967.

12. Commercially available from Aldrich. 